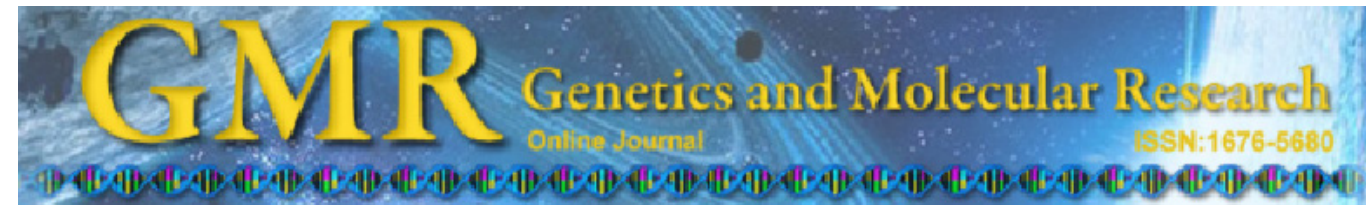

\title{
A new approach for cloning hLIF cDNA from genomic DNA isolated from the oral mucous membrane
}

\author{
Y.H. Cui*, G.Q. Zhu*, Q.J. Chen, Y.F. Wang, M.M. Yang, Y.X. Song, \\ J.G. Wang and B.Y. Cao \\ College of Animal Science and Technology, \\ Northwest Agriculture and Forestry University, Yangling, P.R. China
}

*These authors contributed equally to this study.

Corresponding author: B.Y. Cao

E-mail: caobinyun@yahoo.com.cn

Genet. Mol. Res. 10 (4): 3455-3462 (2011)

Received December 7, 2010

Accepted October 8, 2011

Published November 25, 2011

DOI http://dx.doi.org/10.4238/2011.November.25.2

\begin{abstract}
Complementary DNA (cDNA) is valuable for investigating protein structure and function in the study of life science, but it is difficult to obtain by traditional reverse transcription. We employed a novel strategy to clone human leukemia inhibitory factor (hLIF) gene cDNA from genomic DNA, which was directly isolated from the mucous membrane of mouth. The hLIF sequence, which is $609 \mathrm{bp}$ long and is composed of three exons, can be acquired within a few hours by amplifying each exon and splicing all of them using overlap-PCR. This new approach developed is simple, time- and cost-effective, without RNA preparation or cDNA synthesis, and is not limited to the specific tissues for a particular gene and the expression level of the gene.
\end{abstract}

Key words: hLIF; cDNA cloning; Oral mucous membrane;

Overlap PCR 


\section{INTRODUCTION}

In the field of molecular biology, cDNA cloning is one of the most frequently used technologies, and is the first and essential step in the genetic manipulation of mammals. The traditional method to obtain the cDNA fragment is by reverse transcriptase PCR amplification (RT-PCR). In short, a reverse transcriptase reverse transcribes the RNA into cDNA, and then the acquired cDNA is used as a template for subsequent PCR with primers designed according to the target gene (Bertrand et al., 1994). But the cDNA is difficult to acquire, because of the harvest of tissues that express the gene of interest, mRNA preparation and reverse transcription. And since some target genes are lowly expressed in some specific tissues, their mRNA is difficult to obtain. What's more, RNases, which can degrade the mRNA, are very stable and difficult to remove completely, so special consumable material must be used when dealing with RNA (Bustin and Nolan, 2004). The cDNA of the target gene is still difficult to obtain even though some methods have been proposed to prevent RNA degradation and optimize RNA storage conditions (Eikmans et al., 2000). It is clear that cDNA cloning is susceptible to some challenges.

As is well known, gene sequences contain exons and introns, which exist alternately, and the exons can reverse-transcribe into cDNA. So overlap-extension PCR (Young and Dong, 2004) and assembly PCR (Hoover et al., 2002; Xiong et al., 2006) can be used to synthesize the cDNA of the target gene. Assembly PCR was originally adopted in DNA shuffling for in vitro evolution of DNA molecules (Stemmer, 1994a,b). As the genomic DNA sequence and databases have been perfected, it has become easier to acquire information interrelated to any gene. However, cDNA cloning of bioactive cytokine directly from genomic DNA has rarely been reported, because these cytokines have just been observed in specific tissues, like the hLIF that exists in human blood.

Here, we report a novel method for synthesizing hLIF cDNA based on overlappingPCR directly from the oral mucous membrane. Briefly, the exons of the hLIF gene are PCRamplified separately by using a primer pair containing homologous sequences with two adjacent exons, which are then spliced together in their spontaneously order.

\section{MATERIAL AND METHODS}

\section{Enzymes, kits and chemicals}

Pfu DNA polymerase, DNA marker I and DH5 $\alpha$ competent cells were acquired from Takara Biotech Co., Ltd. (Dalian, China). RNAprep pure Blood kit, TIANamp swab DNA kit and TIANscript RT Kit were purchased from Tiangen Biotech Co., Ltd. (Beijing, China). pGEM $^{\circledR}$-T easy vector, Wizard SV Gel and PCR Clean-up System were acquired from Promega Biotech Co., Ltd. (Madison, WI). IPTG, X-Gal and other chemicals were acquired from BaoXin Scientific Equipment Co., Ltd. (Yangling, China).

\section{Primer design}

The human leukemia inhibitory factor (hLIF) mRNA in the NCBI Genbank database was retrieved, and the Genbank accession number NM_002309.3 was obtained and submitted using this program. The primer sequences of the overlapping PCR and RT-PCR are listed in Table 1. Oligonucleotides were synthesized and purified by Genscript Co., Ltd. (Nanjing, China). 


\begin{tabular}{|c|c|c|}
\hline Exons & Primers & Sequence for primers \\
\hline \multirow[t]{2}{*}{2} & $\mathrm{P} 1$ & 5'-ATGAAGGTCTTGGCGGCAG-GAGTTGTGCCCCTGCTGTTG-3' \\
\hline & $\mathrm{P} 2$ & 5'-CCCCTGGGCTGTGTAATAGAGAATAAAGAGGGCATTGG-3' \\
\hline \multirow[t]{2}{*}{3} & P3 & 5'-СТCTTTATTCTCTAT-TACACAGCCCAGGGGGAGCCG-3' \\
\hline & $\mathrm{P} 4$ & 5'-GCTAGAAGGCCTGGGCCAACACGGCG-3' \\
\hline
\end{tabular}

The sequence in the box is the coding sequence (19 bp) of the first exon, added in the primer P1 like the restriction enzyme sites. The 30 bases underlined are the overlapping region at the exon-exon joining sites, which made it is more easy to splicing the exons.

\section{Isolation of total RNA and genomic DNA}

Total RNA of whole blood collected from a healthy honor was extracted with RNAprep pure Blood kit (TIANGEN) according to manufacturer instructions; the genomic DNA was extracted with a TIANamp swab DNA kit (TIANGEN) from the oral mucous membrane. The purified RNA or DNA was dissolved in $30 \mu \mathrm{L}$ and its concentration was determined by a spectrophotometer-ND-1000.

\section{cDNA clone of hLIF using overlap-PCR}

First, the optimal annealing temperature was determined by gradient PCR, and then completed exon amplification. For each exon, a standard PCR was performed with the following $50 \mu \mathrm{L}$ reaction mixture (Table 2), and Exon amplification was completed in individual tubes on a Bio-RAD PCR system using the following thermal cycling conditions (Table 3).

\begin{tabular}{lc} 
Table 2. The reaction mixture of PCR amplification. \\
\hline Components & Volume \\
\hline 10X Pfu Buffer with $\mathrm{MgSO}_{4}$ & $5 \mu \mathrm{L}$ \\
dNTP mix, 2 mM each & $5 \mu \mathrm{L}$ \\
Forward primer & $1.5 \mu \mathrm{L}$ \\
Reverse primer & $1.5 \mu \mathrm{L}$ \\
Template DNA & $4 \mu \mathrm{L}$ \\
Pfu DNA polymerase & $0.6 \mu \mathrm{L}$ \\
Water, nuclease-free & up to $50 \mu \mathrm{L}$ \\
\hline
\end{tabular}

\begin{tabular}{lccc} 
Table 3. The conditions of PCR amplification. & & \\
\hline Step & Temperature ${ }^{\circ} \mathrm{C}$ & Time & Number of cycles \\
\hline Initial denaturation & 95 & $3 \mathrm{~min}$ & 1 \\
Denaturation & 95 & $30 \mathrm{~s}$ & 30 \\
Annealing & 58 & $30 \mathrm{~s}$ & $30 \mathrm{~s}$ \\
Extension & 72 & $8 \mathrm{~min}$ & 1 \\
Final extension & 72 &
\end{tabular}

The PCR products were purified and mixed, and used as a template in the overlap-PCR that followed. In the overlapping PCR, the primers were the forward primer of the second exon and the reverse primer of the third, and the other components were the same as above. Except for adding $45 \mathrm{~s}$ to the extension time, there was no difference with the above. And then the full- 
length ORF sequence was amplified and all the PCR products were subjected to $1.5 \%(\mathrm{w} / \mathrm{v})$ agarose gel electrophoresis and the gel containing appropriate DNA segments were excised and the segments were purified with Wizard SV Gel and PCR Clean-up System (Promega).

\section{The hLIF clone by RT-PCR}

The cDNA by TIANscript RT Kit was used as the template in the RT-PCR, and the PCR components and parameters were the same as the above.

\section{Cloning and sequencing}

The resultant hLIFs were cloned into pGEM $^{\circledR}$-T easy vector, respectively. DNA sequencing was carried out by the Genscript Company.

\section{RESULTS}

\section{cDNA clone of hLIF using overlap-PCR}

Exon 2 and exon 3 of hLIF were amplified using standard PCR, and exon 2 contained a 19 bp coding sequence of exon1, and the 30 base overlapping region was taken into each exon following the exon amplification, which made it easier to splice the exons. Then the primers P1 and P4 were used to perform another universal PCR to splice the exons. The PCR product and two of the exons are shown on 1.5\% agarose gel electrophoresis (Figure 1). The predicted length of exon2, exon 3 and the hLIF gene are 213, 426 and 609 bp respectively. These segments were sequenced and blasted with the Genbank database, and the results indicated that the coding sequence of the hLIF was harbored correctly. And the two separate exons were successfully spliced. There was an average of 0.5 point mutations per clone, which were silent mutations (Figure $3 \mathrm{~A}$ ).

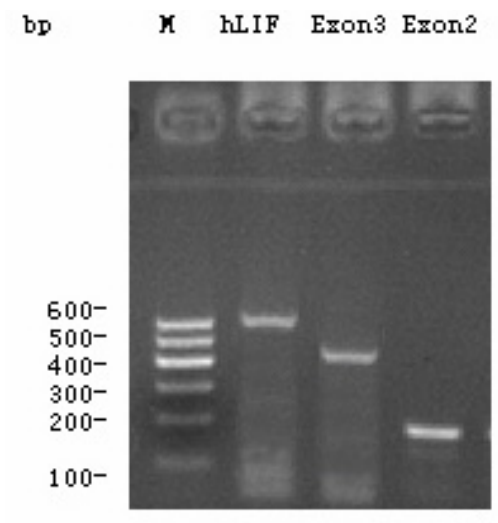

A

Figure 1. Amplification and splicing of hLIF coding sequence by exon-splicing. "M" is DNA marker I. The figure 1 showed that the exons were amplified by the standard PCR with the exon own primers and the hLIF was performed by the overlapping PCR with P1, P4. The length of the performed fragments of exon2, exon3 and the full-length hLIF is $213,426,609$ bp respectively. 


\section{The hLIF clone by RT-PCR}

We performed RT-PCR to obtain the coding sequence of the hLIF gene, which was demonstrated on $1.5 \%$ agarose gel electrophoresis (Figure 2). DNA sequencing of the resultant hLIFs was carried out by the Genscript Company. The mutation rate was higher in the hLIF gene by reverse transcription as compared to overlap-PCR, and the mutations resulted in changes to amino acids, even ORFs. The results are shown in Figure 3B of the blast with the Genbank database.

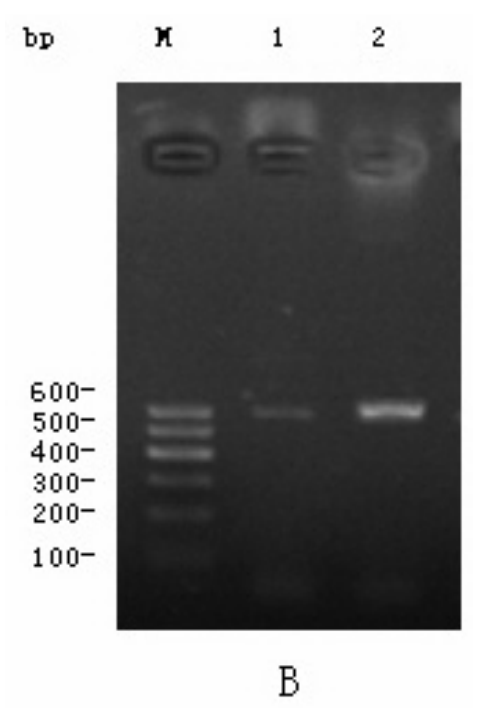

Figure 2. Amplification of hLIF coding sequence by RT-PCR. In the figure 2, the coding sequence of hLIF gene was successfully reverse transcribed and amplified, which is $609 \mathrm{bp}$ (lane 1), and lanes 2 was the full-length hLIF by exon-splicing. The figure indicated that it is more easily to obtain the coding sequence of target gene by exonsplicing in terms of time and quantity, compared with the traditional reverse transcription. "M" is DNA marker I.

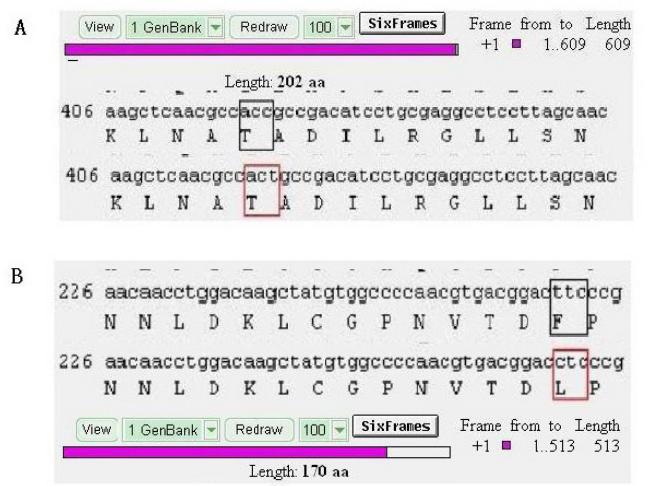

Figure 3. The results blasted with Genbank database. A. The cDNA of hLIF obtained using the overlap PCR. The length of ORF of hLIF is $609 \mathrm{bp}$, which is correct. Even though the $\mathrm{C}$ become to T, the amino acid is unchanged, and that is the silent mutation. B. The resultant hLIF cDNA by RT-PCR. The figure indicated the amino acid and the ORF were changed because there existed the mutation. 


\section{DISCUSSION}

The gene synthesis based PCR is now used in molecular biology laboratories worldwide, with free online tools that automate primer design and optimize the codon usage for efficient expression in the target organism. Here we reported a new gene synthesis strategy, "PCR-based genomic exon splicing", which takes advantage of genomic DNA to avoid mRNA preparation and reverse transcription.

In traditional RT-PCR, the RNA must be extracted, and the gene comes from humans, so we must consider how to collect the sample. For those reasons, it is most reasonable that a five microliter whole blood sample was collected from a healthy donor. Total RNA was then extracted according to manufacturer instructions of the appropriate kit. And in that processing, we must use special consumable material to overcome the RNases. Thus we acquired the genomic DNA using a TIANamp swab DNA kit, which only swabs in the oral membrane; then according to manufacturer instructions, the genomic DNA was easily extracted within 90 min. So, the method to obtain the genomic DNA is simple, rapid, reliable and stable and omits the complex process when extracting RNA.

In the assembly PCR experiment using Tap polymerase, which is error-prone, an average of 3 point mutations was found in a $720 \mathrm{bp}$ synthesis sequence and the mutation rate was $0.4 \%$ (Zhu et al., 2007). It was reported that there is a point mutation rate of $0.1 \%$ for using the proof reading thermo-stable DNA polymerase Pfu (Stemmer et al., 1995). There are reports that the mutation rate was reduced to about $0.01 \%$ when the Phusion high-fidelity DNA polymerase was used alone, and no point mutation was identified when the T7 endonuclease I was used in combination with Phusion high-fidelity DNA polymerase (An et al., 2007).

So we used the Pfu DNA polymerase to perform hLIF cDNA cloning to avoid the unwanted point mutations and enhance fidelity. Our results showed that there was scarcely a point mutation in the $610 \mathrm{bp}$ synthesis sequence in the new gene synthesis strategy, "PCRbased genomic exons splicing". However we found an average of 2 point mutations per clone in the target sequence when the hLIF cDNA was obtained by reverse transcription. This clearly demonstrated that the fidelity was higher and the mutation rate was lower when the new gene synthesis strategy was used.

Vandenbroeck et al. (1991) reported a PCR-based exon ligation, which required multiple rounds of PCR and multiple ligation reactions of blunt-end PCR products and restriction fragments. An in vitro exon ligation method was proposed by Lebedenko et al., and the entire process includes two-step PCR to amplify the exons, cloning of all exons separately, and introduction of specific restriction enzymes and ligation of multiple restriction fragments (Lebedenko et al., 1991). An et al. proposed a novel PCR mediated Genomic DNA Splicing method (GDS strategy) for cloning of any eukaryotic cDNA or coding sequence from a genomic DNA preparation (An et al., 2007), but it required that the exon primers, overlapping primers and outmost primers be designed separately. And there was a method proposed for cDNA synthesis based upon the use of the class IIS restriction enzymes to acquire the coding sequence of the Hoxa7 gene, so the matching restriction enzymes must be taken into account at the exon-exon joining sites (Li et al., 2008). And due to the specificity of the gene, the coding sequence ( $19 \mathrm{bp}$ ) of the first exon can be spliced to the second exon fragment in the form of the deliberately designed primers, like adding the restriction enzyme sites. Although the coding sequence of hLIF gene is composed of only three exons and the coding sequence in 
the first exon is only $19 \mathrm{bp}$, two pairs of overlapping primers have to be designed. The $19 \mathrm{bp}$ coding sequence in the first exon should be designed in the primer by adding the recognition sequence of the incision enzyme. Then the cDNA sequence (only $19 \mathrm{bp}$ ) of the first exon can be connected to other spliced fragments.

As compared with reverse transcription PCR, the new gene synthesis strategy in this report has the following advantages: the most attractive one is that the laborious RNA preparation and reverse transcription procedure can be omitted, saving time and energy, and the expense of RNA extraction kits and cDNA synthesis kits. Also, it might be more accurate since it omits the reverse transcription which may increase the probability of mismatched nucleotide incorporation because the M-MLV does not possess 3'-5' exonuclease-dependent proofreading activity. The second one is that the target DNA sequence obtained is high fidelity and the process is simple, easy and very efficacious. Only a few hours is needed for the whole splicing process, which employs two rounds of PCR amplification. Moreover there is no point mutation and the fidelity is rather high. And we only designed a pair of oligonucleotide primers for one exon, which can omit a round of PCR amplification and sharply limit the mismatch of PCR. The third concern about our gene synthesis strategy is that it is not necessary to consider the expression level, the quantity of mRNA or any specific tissue for a particular target gene. And because genomic DNA is more stable than RNA, it may be more practical to clone many genes and synthesize cDNA sequences.

In conclusion, with a new gene synthesis strategy, "PCR-based genomic exons splicing", we successfully acquired the full-length 609 bp coding sequence of the hLIF gene, which consists of three exons. The approach is simple, rapid, high fidelity, highly efficient and costeffective without RNA preparation or cDNA synthesis, and does not consider tissue specificity and the expression of the target gene, which can connect with two or more unassociated segments without restriction enzymes or DNA ligase.

\section{ACKNOWLEDGMENTS}

Research supported by the National 863 Program of China (\#2007AA10Z167).

\section{REFERENCES}

An X, Lu J, Huang JD, Zhang B, et al. (2007). Rapid assembly of multiple-exon cDNA directly from genomic DNA. PLoS One 2: e1179.

Bertrand E, Pictet R and Grange T (1994). Can hammerhead ribozymes be efficient tools to inactivate gene function? Nucleic Acids Res. 22: 293-300.

Bustin SA and Nolan T (2004). Pitfalls of quantitative real-time reverse-transcription polymerase chain reaction. $J$. Biomol. Tech. 15: 155-166.

Eikmans M, Baelde HJ, De HE and Bruijn JA (2000). Processing renal biopsies for diagnostic mRNA quantification: improvement of RNA extraction and storage conditions. J. Am. Soc. Nephrol. 11: 868-873.

Hoover DM and Lubkowski J (2002). DNAWorks: an automated method for designing oligonucleotides for PCR-based gene synthesis. Nucleic Acids Res. 30: e43.

Lebedenko EN, Birikh KR, Plutalov OV and Berlin Y (1991). Method of artificial DNA splicing by directed ligation (SDL). Nucleic Acids Res. 19: 6757-6761.

Li XX, Zheng F, Jiao YL, Guo G, et al. (2008). An alternative approach to synthesize cDNA bypassing traditional reverse transcription. Mol. Biotechnol. 39: 201-206.

Stemmer WP (1994a). DNA shuffling by random fragmentation and reassembly: in vitro recombination for molecular evolution. Proc. Natl. Acad. Sci. U. S. A. 91: 10747-10751. 
Stemmer WP (1994b). Rapid evolution of a protein in vitro by DNA shuffling. Nature 370: 389-391.

Stemmer WP, Crameri A, Ha KD, Brennan TM, et al. (1995). Single-step assembly of a gene and entire plasmid from large numbers of oligodeoxyribonucleotides. Gene 164: 49-53.

Vandenbroeck K, Dijkmans R, van Aerschot A and Billiau A (1991). Engineering by PCR-based exon amplification of the genomic porcine interferon-gamma DNA for expression in Escherichia coli. Biochem. Biophys. Res. Commun. 180: $1408-1415$.

Xiong AS, Yao QH, Peng RH, Duan H, et al. (2006). PCR-based accurate synthesis of long DNA sequences. Nat. Protoc. 1: 791-797.

Young L and Dong Q (2004). Two-step total gene synthesis method. Nucleic Acids Res. 32: e59.

Zhu X, Chen J, Zhang X, An X, et al. (2007). Correction of a mutation in a synthetic gene by DREAM technique, a sitedirected mutagenesis. China Biotechnol. 27: 86-92. 